\title{
Unilateral Pulmonary Hypoplasia in a Child
}

\author{
Gourab Dewan' \\ 'Department of Internal Medicine, Rangamati General Hospital, Rangamati, Bangladesh.
}

\section{ABSTRACT}

Pulmonary hypoplasia is an uncommon congenital anomaly. A case reported in a six year old male child from Bangladesh who presented with chronic dry cough, episodic fever and occasional haemoptysis causing confusion with tuberculosis. X-ray suggested lung collapse. Final diagnosis reached by combined bronchoscopy, computed tomogram scan of chest and pulmonary angiogram. In a child with complete radiological lung collapse possibility of pulmonary hypoplasia should be kept in mind.

Keywords: Bangladesh; child; congenital anomalies; pulmonary hypoplasia.

\section{INTRODUCTION}

Congenital pulmonary anomalies are rare in practice. Pulmonary hypoplasia a condition in which there is reduction in number or size of important intrapulmonary structures like bronchi and its branches, vascular territories and alveoli. ${ }^{1}$ Gross morphology of effected lung remains normal apart from reduction in size and lung volume. Effected lung become fibrosed and nonfunctioning. Pulmonary hypoplasia can be primary or secondary in origin. Secondary cases in $60 \%$ show fetal or maternal abnormality, rests include intrathorasic mass lesion or chest wall deformities. ${ }^{2}$ The condition may be accompanied by congenital abnormalities involving other organs. It sometimes mimics radiologically more common pulmonary conditions.

\section{CASE REPORT}

A six year old boy presented with one year history of recurrent dry cough, fever and occasional haemoptysis. The boy weighted $14 \mathrm{Kg}$ and $109 \mathrm{~cm}$ in height. BMI was 11.8 which was less than $3^{\text {rd }}$ percentile for a child of his age (underweight). He had visible chest wall deformity on left (Figure 1) and reduced chest expansion, dull percussion note, diminished breath sound on same side. $X$-ray revealed an opaque left hemithorax with shift of mediastinum to left suggesting total lung collapse (Figure 2). Sputum for acid fast bacilli was negative. Blood count within normal limit. A provisional diagnosis of tubercular lymphadenopathy associated lung collapse was in mind. Bronchoscopy ordered next. It showed narrowing at end of left principal bronchus and could not gain access to upper and lower lobe bronchus. Right side was normal. Computed tomogram (CT) scan showed marked thorasic asymmetry and herniation of right lung to left hemithorax with heart placed at extreme left of chest cavity. Only proximal part of left principal bronchus could be delineated clearly (Figure 3 ). A heterogenous soft tissue density was located behind heart in lower part of left hemithorax (rudimentary lung tissue). No mediastinal lymphadenopathy or abnormal mass lesion was seen. CT angiogram showed grossly hypoplastic main pulmonary vessels and its branches on left side (Figure 4). No additional congenital anomalies detected in other systems of the patient. A diagnosis of unilateral primary pulmonary hypoplasia was made.

Correspondence: Dr. Gourab Dewan, Department of Internal Medicine, Rangamati General Hospital, Rangamati, Bangladesh. Email: gourab.dewan@yahoo.com, Phone: +88-01712599770. 

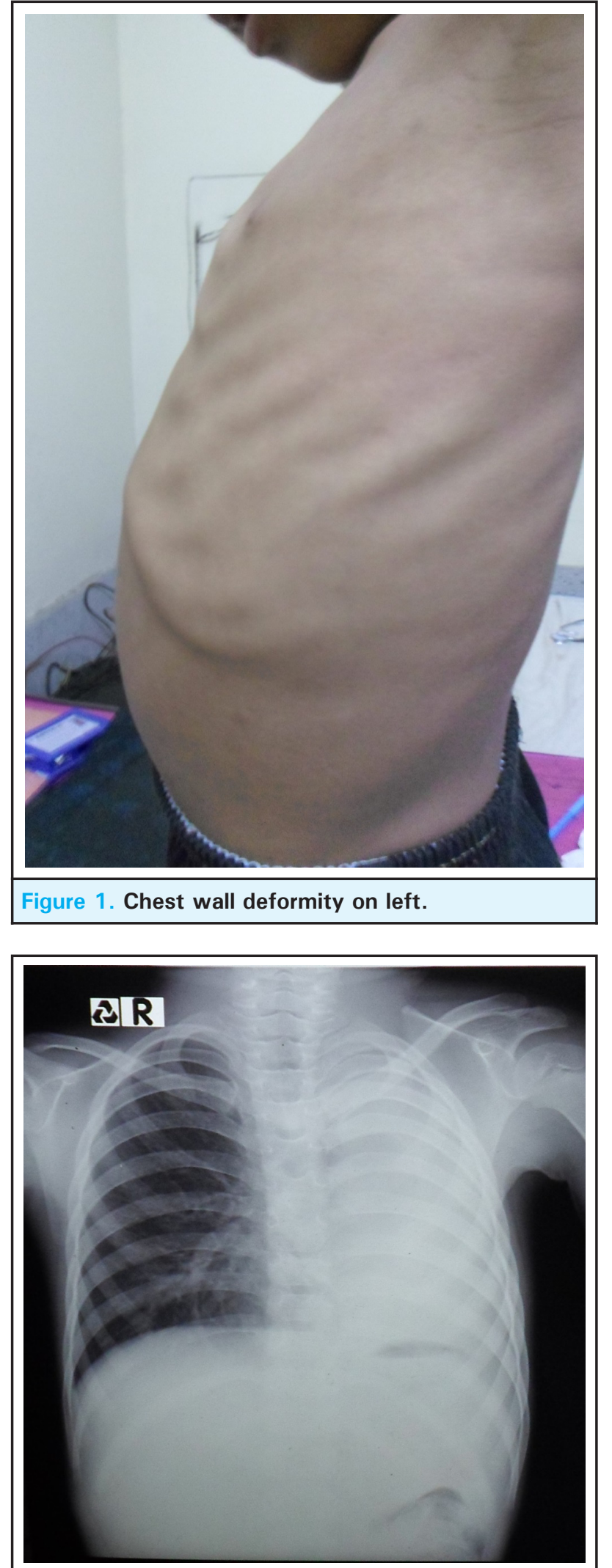

Figure 2. An opaque left hemithorax with mediastinal shift to left.

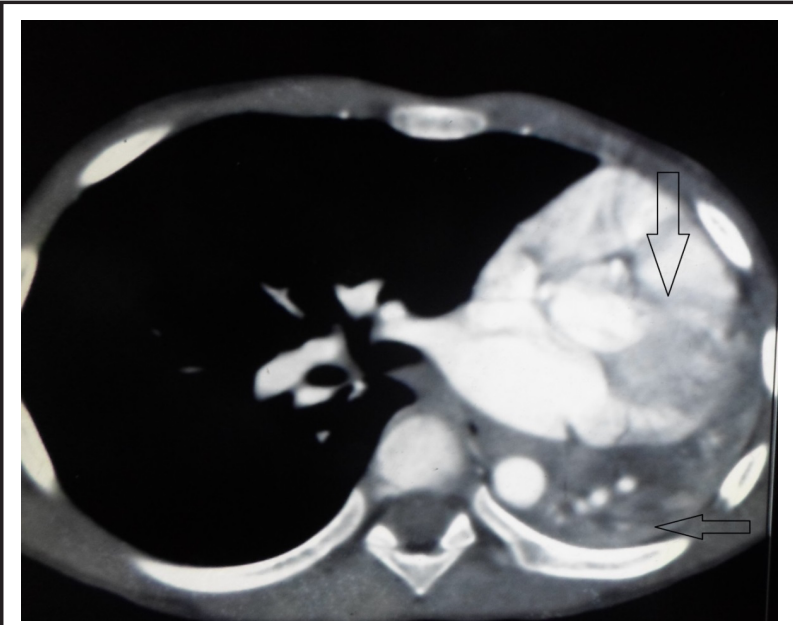

Figure 3. CT scan of chest showing right lung and heart (down arrow) shifted to left hemithorax.

Rudimentary left lung visible as heterogenous soft tissue (horizontal arrow) behind heart.

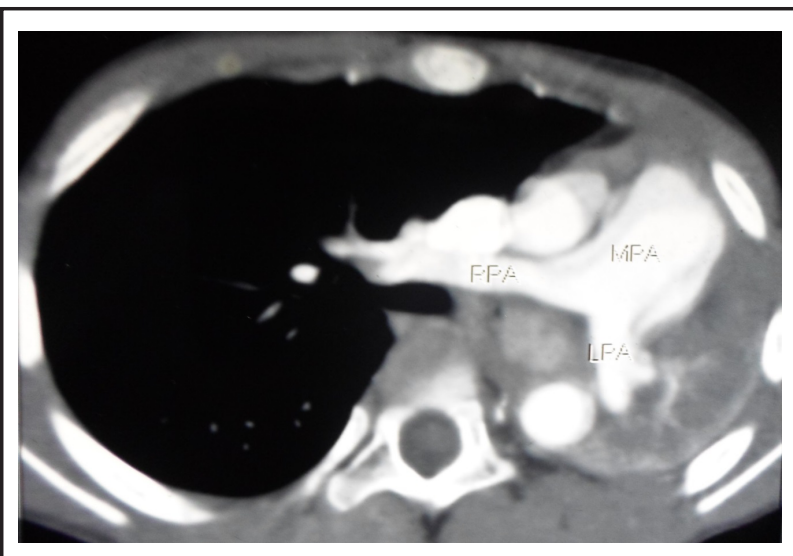

Figure 4. CT pulmonary angiogram showing small left pulmonary artery arising from pulmonary trunk, $($ MPA $=$ Main pulmonary artery, RPA $=$ Right pulmonary artery, LPA $=$ Left pulmonary artery).

\section{DISCUSSION}

Pulmonary hypoplasia is usually unilateral and occasionally reported to be bilateral. Reported frequency of pulmonary hypoplasia is $1.4 \%$ of all births by Knox et al. ${ }^{3}$ However Gulen et al. and Kant estimates occurrence to be 1-2 cases per 12000 births..$^{4,5}$ Development of bronchial tree takes place during $26^{\text {th }}$ to $31^{\text {st }}$ day of intrauterine life. ${ }^{5}$ And it is between fourth to $24^{\text {th }}$ week that pulmonary malformations take place. ${ }^{2}$ After Boyden, three degrees of pulmonary developmental abnormalities are described as: 1) Pulmonary agenesis (total absence of the lung tissue), 2) Pulmonary aplasia (lung tissue absent, only a rudimentary bronchus) and 3) Pulmonary hypoplasia (underdeveloped lung tissue). ${ }^{6}$ Monaldi categorized pulmonary congenital anomalies 
Dewan Unilateral Pulmonary Hypoplasia in a Child

in to four groups. Group I: absent tracheal bifurcation; Group II: rudimentary main bronchus; Group III: arrested development after bifurcation of the main bronchus; Group IV: Incomplete development of intralobar segments of bronchi. ${ }^{7}$ Current patient falls to Monaldi group IV and Boyden's third degree developmental anomaly.

Pathologically there is usually $50-75 \%$ reduction in bronchial tree and reduction of alveoli number by one third along with reduction in size. ${ }^{5}$ Associations include malformation involving heart (tetralogy of Fallot's), gut, kidney, diaphragm, central nervous system (Anencephaly, hydrocephalus), musculoskeletal system and Down's syndrome. ${ }^{5}$

Presentation may be as soon as birth up to very old age. Time of presentation, severity of symptoms depends on degree of underlying abnormality. Most reported patients are newborn or infant. Respiratory distress within five minutes of birth is typical for these patients. ${ }^{4}$ However asymptomatic cases until adulthood has been reported. ${ }^{1}$ Common respiratory symptoms are cough, dyspnea, haemoptysis, chest pain or recurrent fever due to respiratory tract infection. Present patient resembled pulmonary tuberculosis in clinical manifestation similar to the patient reported by kant. ${ }^{5}$

Chest X-ray is usually the primary investigation done. It is not diagnostic and initiates series of follow up investigations. It generally shows features of volume reduction eg rib crowding, densely opaque hemithorax and mediastinal shift. Diagnosis in life is imaging based. Investigations helpful to diagnose this condition include bronchoscopy, bronchography, CT scan of chest, magnetic resonance imaging (MRI), pulmonary
CT angiography (vascular hypoplasia or aberration). CT scans show an abnormally shaped thorax, volume reduction on the affected side, compensatory hyperinflation of the opposite side, ipsilateral mediastinal shift, hypoplastic airways, and rib abnormalities. ${ }^{1-4,7}$ CT also excludes diaphragmatic hernia or presence of mass lesion that might lead to atelectasis of lung. An effort should be taken to look for possible congenital anomalies in other systems. Current patient had no abnormalities in other systems. MRI and ultrasonogram can be used to predict hypoplasia in fetus. ${ }^{8}$

Patient or parents need to be addressed about the nature of underlying disorder and possible consequences. Treatment in this age of current patient consists of treatment of recurrent respiratory infection, use of expectorant, bronchodilator. Full vaccination against influenza, pneumococci and respiratory syncytial virus must. ${ }^{5}$ Pneumonectomy may be considered. Surgery some times helpful in case of diaphragmatic hernia. Differential diagnosis includes lung collapse, Swyer James syndrome, Scimitar syndrome and Poland syndrome.

Prognosis in those with left sided hypoplasia reported to be better as right lung with its three lobes can minimize the shortcomings by compensatory hyperinflation. ${ }^{1}$ Degree of lung and co-organ involvement often influences prognosis. Primary variety has better outcome since patients lack additional abnormalities. ${ }^{1}$ Those presenting in neonatal period has high mortality rate.

In conclusion rare possibility of pulmonary hypoplasia should be kept in mind while assessing a case of radiologically opaque hemithorax.

\section{REFERENCES}

1. Georgescu A, Nuta C, Bondari S. 3D Imaging in Unilateral Primary Pulmonary Hypoplasia in an Adult: A Case Report. Case Reports in Radiology. 2011;2011:659586.

2. Albay S, CankaL F, Tunali S, Ozan H. Unilateral pulmonary hypoplasia. International Journal of Anatomical Variations. 2008;1:23-5.

3. Knox WF, Barson AJ. Pulmonary hypoplasia in a regional perinatal unit. Early Hum Dev. 1986; 14(1):33-42.

4. Gülen F, Kar S, Midyat L, Demir E, Özyurt S, Özyürek AR, Tanaç R, Ceylan N. A rare clinical presentation of pulmonary hypoplasia. Open Journal of Pediatrics. 2011;1:75-8.
5. Kant S. Unilateral pulmonary hypoplasia - a case report. Lung India. 2007;24:69-71

6. Boyden EA. Developmental anomalies of the lungs. Am J Surg. 1955; 89: 79-89.

7. Monaldi, V. Malformative bronchopulmonary diseases caused by anatomical defects. Minerva Medica. 1960;51:3474-8.

8. Tanigaki S, Miyakoshi K, Tanaka M, et al. Pulmonary hypoplasia: prediction with use of ratio of MR imaging-measured fetal lung volume to US-estimated fetal body weight. Radiology. 2004; 232(3):767-72. 